\title{
Approximate Solution for Nonlinear System of Integro-Differential Equations of Volterra Type with Boundary Conditions
}

\author{
Ghada Shuker Jameel \\ Department of Mathematics / College of Education \\ University of Mosul, Mosul Iraq
}

Received

07 / 11 / 2013
Accepted

16 / 02 / 2014

\begin{abstract}
الخلاصة
يتضمن البحث دراسة تقارب الحل لنظام من المعادلات التكاملية-التفاضلية اللاخطية

من نوع فولتيرا ذات شـروط حدوديـة، وذللك بالاعتمـاد على الطريقـة التحليليـة-العدديـة لدراسـة

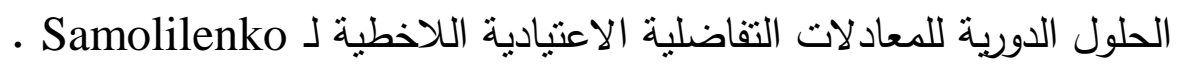

\section{ABSTRACT}

In this study we investigate the approximation of the solution for nonlinear system of integro-differential equations of Volterra type with boundary conditions.

The numerical-analytic method of periodic solutions for ordinary differential equations of Samoilenko has been used of this work.

\section{Introduction}

The approximate periodic solutions for nonlinear systems of integro-differential equations have been used to study in many problems $[1,2,3,4,5]$.

Ghada [2], used the method above to investigate the approximate periodic solution for nonlinear system of integro-differential equations of Volterra type which has the form:- 


$$
\frac{d x(t)}{d t}=A(t) x(t)+\int_{0}^{t} K(t, s) F(t, s, x) d s+f(t)
$$

Also these investigations lend us to the improving and extending some work of Ghada [2]. equation:

Consider the following system of nonlinear integro-differential

$$
\frac{d x(t)}{d t}=A(t) x(t)+\int_{0}^{t} K(t, s) F(t, s, x) d s+f(t),
$$

with boundary conditions

$$
B x(0)+C x(T)=d
$$

Here $x \in G \subseteq R^{n}, G$ is a closed and bounded domain subset of Euclidean spaces $R^{n}$.

Let the vectors functions:

$$
\begin{aligned}
& f(t)=\left(f_{1}(t), f_{2}(t), \ldots, f_{n}(t)\right) \\
& F(t, s, x)=\left(F_{1}(t, s, x), F_{2}(t, s, x), \ldots, F_{n}(t, s, x)\right),
\end{aligned}
$$

where the functions $F(t, s, x)$ and $f(t)$ are continuous, bounded on the domain:

$$
(t, s, x) \in[0, T] \times[0, T] \times G,
$$

where $B=\left(B_{i j}\right), C=\left(C_{i j}\right)$ are constants positive matrices $(n \times n)$.

Suppose that the functions $F(t, s, x)$ and $f(t)$ satisfies the following inequalities:

$$
\begin{aligned}
& \|F(t, s, x)\| \leq M \quad, \quad\|f(t)\| \leq N \\
& \left\|F\left(t, s, x_{1}\right)-F\left(t, s, x_{2}\right)\right\| \leq L\left\|x_{1}-x_{2}\right\|
\end{aligned}
$$

for all $t \in[0, T], s \in[0, T]$ and $x, x_{1}, x_{2} \in G$, where $M, N$ and $L$ are positive constants.

Let $A(t), K(t, s)$ are $(n \times n)$ non-negative matrices which is defined and continuous on (1.3), periodic in $t$ of period $\mathrm{T}$, provided that:

$$
\begin{aligned}
& \|K(t, s)\| \leq H \\
& \left\|\int^{0} A(\eta) d \eta\right\| \leq Q
\end{aligned}
$$

where $-\infty<0 \leq s \leq t \leq T<\infty$ and $Q, H$ are a positive constants.

We define the non-empty sets as follows: 
$G_{f}=G-\frac{T}{2} M_{1}+\beta$

where $M_{1}=Q[H M T+N],\|\cdot\|=\max _{t \in[0, T]}|\cdot|$ and $\beta=\frac{t}{T} Q\left[\left(C^{-1} A+E\right) x_{0}-C^{-1} d Q^{-1}\right]$.

Furthermore, we suppose that:

$q=\left[(Q H L T) \frac{T}{2}\right]<1$

By using lemma 3.1[5], we can state and prove the following lemma.

\section{Lemma 1.1}

Let $f(t)$ and $F(t, s, x)$ be continuous vector functions on the interval $[0, T]$ then the following:

$\| \int_{0}^{t} \int^{t} A(\eta) d \eta\left[\int_{0}^{s} K(s, \tau) F\left(s, \tau, x\left(\tau, x_{0}\right)\right) d \tau+f(s)\right] d s-\frac{1}{T} \int_{0}^{t} \int^{t} A A(\eta) d \eta\left[\left(c^{0} A+E\right) x_{0}-c^{-1} d e^{-\int_{0}^{t} A(\eta) d \eta}\right] d s-$ $-\frac{1}{T} \int_{0}^{t} \int_{0}^{T} e^{t} A(\eta) d \eta\left[\int_{0}^{s} K(s, \tau) F\left(s, \tau, x\left(\tau, x_{0}\right)\right) d \tau+f(s)\right] d t d s \| \leq \alpha(t) M_{1}+\beta$

Satisfying for $\quad 0 \leq t \leq T$ and $\quad \alpha(t) \leq \frac{T}{2} \quad$ where $\quad \alpha(t)=2 t\left(1-\frac{t}{T}\right)$, $M_{1}=Q[H M T+N]$ and $\beta=\frac{t}{T} Q\left[\left(C^{-1} A+E\right) x_{0}-C^{-1} d Q^{-1}\right]$.

proof:

$\int_{0}^{t} e^{t} A(\eta) d \eta\left[\int_{0}^{s} K(s, \tau) F\left(s, \tau, x\left(\tau, x_{0}\right)\right) d \tau+f(s)\right] d s-\frac{1}{T} \int_{0}^{t} \int^{t} A(\eta) d \eta\left[\left(c^{-1} A+E\right) x_{0}-c^{-1} d e^{-\int_{0}^{t} A(\eta) d \eta}\right] d s-$ $-\frac{1}{T} \iint_{0}^{t} \int_{0}^{T} e^{t} A(\eta) d \eta\left[\int_{0}^{s} K(s, \tau) F\left(s, \tau, x\left(\tau, x_{0}\right)\right) d \tau+f(s)\right] d t d s \|=$

$=\| \int_{0}^{t} e^{t} A(\eta) d \eta\left[\int_{0}^{s} K(s, \tau) F\left(s, \tau, x\left(\tau, x_{0}\right)\right) d \tau+f(s)\right] d s-\frac{t}{T} e^{t} A(\eta) d \eta\left[\left(c^{-1} A+E\right) x_{0}-c^{-1} d e^{-\int_{0}^{t} A(\eta) d \eta}\right]-$ $-\frac{t}{T} \int_{0}^{T} e^{\int_{0}^{0} A(\eta) d \eta}\left[\int_{0}^{s} K(s, \tau) F\left(s, \tau, x\left(\tau, x_{0}\right)\right) d \tau+f(s)\right] d t \| \leq$ 


$$
\begin{gathered}
\leq\left\|\left(1-\frac{t}{T}\right)_{0}^{t} \int_{0}^{t} e^{0} A(\eta) d \eta\left[\int_{0}^{s} K(s, \tau) F\left(s, \tau, x\left(\tau, x_{0}\right)\right) d \tau+f(s)\right] d s\right\|+\| \frac{t}{T} e^{0} A(\eta) d \eta\left[\left(c^{-1} A+E\right) x_{0}-c^{-1} d e^{-\int^{0} A(\eta) d \eta}\right]+ \\
+\left\|\frac{t}{T} \int_{0}^{T} e^{t} A(\eta) d \eta\left[\int_{0}^{s} K(s, \tau) F\left(s, \tau, x\left(\tau, x_{0}\right)\right) d \tau+f(s)\right] d s\right\| \leq \\
\leq\left(1-\frac{t}{T}\right) t[Q H M T+Q N]+\frac{t}{T}(T-t)[Q H M T+Q N]+\frac{t}{T} Q\left[\left(c^{-1} A+E\right) x_{0}-c^{-1} d Q^{-1}\right] \\
=2 t\left(1-\frac{t}{T}\right) Q[H M T+N]+\frac{t}{T} Q\left[\left(c^{-1} A+E\right) x_{0}-c^{-1} d Q^{-1}\right] \\
=\alpha(t) M_{1}+\beta
\end{gathered}
$$

\section{Approximate Solution}

The investigation of approximate solution of the problem (1.1) and (1.2) will be introduced by the following theorem:

\section{Theorem 1}

If the system (1.1) with boundary conditions (1.2) defined in the domain (1.3), continuous in $t, x$ and satisfy the inequalities (1.4), (1.5) and (1.6), then the sequence of functions:

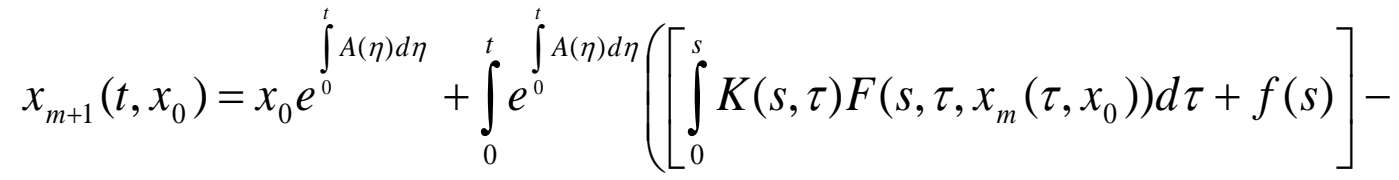

$$
\begin{aligned}
& -\frac{1}{T}\left[\left(c^{-1} A+E\right) x_{0}-c^{-1} d e^{-\int_{0}^{t} A(\eta) d \eta}+\int_{0}^{T}\left[\int_{0}^{s} K(s, \tau) F\left(s, \tau, x_{m}\left(\tau, x_{0}\right)\right) d \tau+f(s)\right] d t\right] d s
\end{aligned}
$$

with

$$
x_{0}\left(t, x_{0}\right)=x_{0} e^{\int^{t} A(\eta) d \eta} \quad, \quad m=0,1,2, \ldots
$$

periodic in $\mathrm{t}$ with period $\mathrm{T}$, converges uniformly when $m \rightarrow \infty$ on the domain:

$$
\left(t, x_{0}\right) \in[0, T] \times G_{f}
$$

to the limit function $x\left(t, x_{0}\right)$ which is satisfying the integral equation: 


$$
\begin{aligned}
x\left(t, x_{0}\right)=x_{0} e^{0} t A(\eta) d \eta & +\int_{0}^{t} e^{t} A(\eta) d \eta\left(\left[\int_{0}^{s} K(s, \tau) F\left(s, \tau, x\left(\tau, x_{0}\right)\right) d \tau+f(s)\right]-\right. \\
& -\frac{1}{T}\left[\left(c^{-1} A+E\right) x_{0}-c^{-1} d e^{-\int_{0}^{t} A(\eta) d \eta}+\int_{0}^{T}\left[\int_{0}^{s} K(s, \tau) F\left(s, \tau, x\left(\tau, x_{0}\right)\right) d \tau+f(s)\right] d t\right] d s
\end{aligned}
$$

its unique solution to (1.1) and satisfies the inequalities:

$$
\begin{aligned}
& \left\|x\left(t, x_{0}\right)-x_{0}\right\| \leq M_{1} \frac{T}{2}+\beta \\
& \left\|x\left(t, x_{0}\right)-x_{m}\left(t, x_{0}\right)\right\| \leq \Lambda^{m}\left(M_{1} \frac{T}{2}+\beta\right)
\end{aligned}
$$

for $t \in[0, T], x_{0} \in G_{f}, \mathrm{~m}=0,1,2, \ldots$

\section{Proof:}

Setting $\mathrm{m}=0$ and using lemma1.1 and the sequence of the functions (2.1) we get:

$$
\begin{aligned}
& \left\|x_{1}\left(t, x_{0}\right)-x_{0}\right\|=\| x_{0} e^{\int_{0}^{t} A(\eta) d \eta}+\int_{0}^{t} e^{t} A(\eta) d \eta\left(\left[\int_{0}^{s} K(s, \tau) F\left(s, \tau, x_{0}\left(\tau, x_{0}\right)\right) d \tau+f(s)\right]-\right. \\
& \left.\left.-\frac{1}{T}\left[\left(c^{-1} A+E\right) x_{0}-c^{-1} d e^{-\int_{0}^{t} A(\eta) d \eta}+\int_{0}^{T}\left[\int_{0}^{s} K(s, \tau) F\left(s, \tau, x_{0}\left(\tau, x_{0}\right)\right) d \tau+f(s)\right] d t\right]-x_{0} e^{0} \int^{t} A(\eta) d \eta\right) d s\right]= \\
& \leq\left(1-\frac{t}{T}\right) t[Q H M T+Q N]+\frac{t}{T}(T-t)[Q H M T+Q N]+\frac{t}{T} Q\left[\left(c^{-1} A+E\right) x_{0}-c^{-1} d Q^{-1}\right] \\
& =2 t\left(1-\frac{t}{T}\right) Q[H M T+N]+\frac{t}{T} Q\left[\left(c^{-1} A+E\right) x_{0}-c^{-1} d Q^{-1}\right] \\
& =\alpha(t) M_{1}+\beta \\
& \left\|x_{1}\left(t, x_{0}\right)-x_{0}\right\| \leq \alpha(t) M_{1}+\beta \leq M_{1} \frac{T}{2}+\beta
\end{aligned}
$$

we get $x_{1}\left(t, x_{0}\right) \in G$, for all $t \in[0, T], x_{0} \in G_{f}$.

By induction we have:

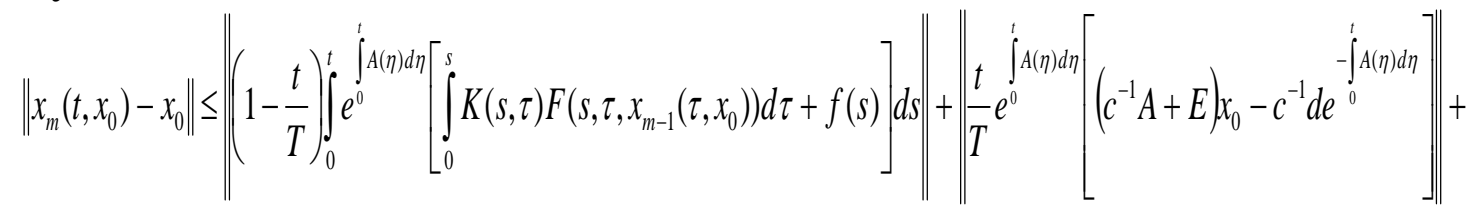

$$
+\left\|\frac{t}{T} \int_{t}^{T} e^{\int^{0} A(\eta) d \eta}\left[\int_{0}^{s} K(s, \tau) F\left(s, \tau, x_{m-1}\left(\tau, x_{0}\right)\right) d \tau+f(s)\right] d s\right\| \leq
$$




$$
\begin{aligned}
& \leq 2 t\left(1-\frac{t}{T}\right) Q[H M T+N]+\frac{t}{T} Q\left[\left(c^{-1} A+E\right) x_{0}-c^{-1} d Q^{-1}\right] \\
& =\alpha(t) M_{1}+\beta
\end{aligned}
$$

where $x_{m}\left(t, x_{0}\right) \in G$, for all $t \in[0, T], x_{0} \in G_{f}$.

We prove now that the sequence (2.1) is uniformly convergent in (2.2). From (2.1), when $\mathrm{m}=1$ we get:

$$
\begin{aligned}
& \left\|x_{2}\left(t, x_{0}\right)-x_{1}\left(t, x_{0}\right)\right\|=\| x_{0} e^{\int_{0}^{t} A(\eta) d \eta}+\int_{0}^{t} e^{t} A(\eta) d \eta\left(\left[\int_{0}^{s} K(s, \tau) F\left(s, \tau, x_{1}\left(\tau, x_{0}\right)\right) d \tau+f(s)\right]-\right. \\
& -\frac{1}{T}\left[\left(c^{-1} A+E\right) x_{0}-c^{-1} d e^{-\int A(\eta) d \eta}+\int_{0}^{T}\left[\int_{0}^{s} K(s, \tau) F\left(s, \tau, x_{1}\left(\tau, x_{0}\right)\right) d \tau+f(s)\right] d t\right] d s- \\
& -x_{0} e^{\int^{0} A(\eta) d \eta}+\int_{0}^{t} e^{t} A(\eta) d \eta\left(\left[\int_{0}^{s} K(s, \tau) F\left(s, \tau, x_{0}\left(\tau, x_{0}\right)\right) d \tau+f(s)\right]+\right. \\
& +\frac{1}{T}\left[\left(c^{-1} A+E\right) x_{0}-c^{-1} d e^{-\int_{0}^{t} A(\eta) d \eta}+\int_{0}^{T}\left[\int_{0}^{s} K(s, \tau) F\left(s, \tau, x_{0}\left(\tau, x_{0}\right)\right) d \tau+f(s)\right] d t\right] d s \| \\
& \leq\left(1-\frac{t}{T}\right) \int_{0}^{t} Q\left[H L T\left(\alpha(t) M_{1}+\beta\right)\right] d s+\frac{t}{T} \int_{t}^{T} Q\left[H L T\left(\alpha(t) M_{1}+\beta\right)\right] d s \\
& \leq \frac{T}{2}(Q H L T)\left(\alpha(t) M_{1}+\beta\right) \\
& =\Lambda\left(\alpha(t) M_{1}+\beta\right)
\end{aligned}
$$

therefore$$
\left\|x_{2}\left(t, x_{0}\right)-x_{1}\left(t, x_{0}\right)\right\| \leq \Lambda\left(M_{1} \frac{T}{2}+\beta\right)
$$

Now when $m=2$ we get the following:

$$
\begin{aligned}
\left\|x_{3}\left(t, x_{0}\right)-x_{2}\left(t, x_{0}\right)\right\| \leq & \left(1-\frac{t}{T}\right) \int_{0}^{t} Q\left[\int_{0}^{s} H L\left\|x_{2}\left(\tau, x_{0}\right)-x_{1}\left(\tau, x_{0}\right)\right\| d \tau\right] d s+ \\
& +\frac{t}{T} \int_{t}^{T} Q\left[\int_{0}^{s} H L\left\|x_{2}\left(\tau, x_{0}\right)-x_{1}\left(\tau, x_{0}\right)\right\| d \tau\right] d s \\
& \leq \frac{T}{2}(Q H L T) \Lambda\left(M_{1} \frac{T}{2}+\beta\right)
\end{aligned}
$$




$$
\left\|x_{3}\left(t, x_{0}\right)-x_{2}\left(t, x_{0}\right)\right\| \leq \Lambda^{2}\left(M_{1} \frac{T}{2}+\beta\right) .
$$

By mathematical induction we have:

$$
\left\|x_{m+1}\left(t, x_{0}\right)-x_{m}\left(t, x_{0}\right)\right\| \leq \Lambda^{m}\left(M_{1} \frac{T}{2}+\beta\right)
$$

for $\mathrm{m}=0,1,2, \ldots$.

By using the condition (1.9), we have

$$
\operatorname{Lim}_{m \rightarrow \infty} \Lambda^{m}=0
$$

So that the rights hand from (2.8) equal zero when $m \rightarrow \infty$. Suppose that $\varepsilon>O$, we get a positive integer $n$ such that $n<m$, and satisfied the next estimation for all $m$ :

$$
\left\|x_{m+p}\left(t, x_{0}\right)-x_{m}\left(t, x_{0}\right)\right\|<\varepsilon, \quad \text { for } P \in N \text {. }
$$

Then according to the definition of uniformly convergent, we find that the sequence $\left\{x_{m}\left(t, x_{0}\right)\right\}_{m=0}^{\infty}$ is uniformly convergent from the function $x\left(t, x_{0}\right)$ and this function be continuous on the same interval.

Putting

$$
\operatorname{Lim}_{m \rightarrow \infty} x_{m}\left(t, x_{0}\right)=x\left(t, x_{0}\right)
$$

Since the sequence of functions $x_{m}\left(t, x_{0}\right)$ is continuous on the domain (2.2) then the limiting function $x\left(t, x_{0}\right)$ is also continues on the same domain.

Also by using lemma1.1 and the relation (2.10), then the inequalities (2.4) and (2.5) are satisfies for all $\mathrm{m}$.

Finally, we show that $x\left(t, x_{0}\right)$ is unique solution of the problem (1.1) and (1.2). On country we suppose that there is at least one different solution $\hat{x}\left(t, x_{0}\right)$ of the problem (1.1) and (1.2), then:

$$
\begin{aligned}
& \hat{x}\left(t, x_{0}\right)=x_{0} e^{\int_{0}^{t} A(\eta) d \eta}+\int_{0}^{t} e^{t} A(\eta) d \eta\left(\left[\int_{0}^{s} K(s, \tau) F\left(s, \tau, \hat{x}\left(\tau, x_{0}\right)\right) d \tau+f(s)\right]-\right. \\
& -\frac{1}{T}\left[\left(c^{-1} A+E\right) x_{0}-c^{-1} d e^{-\iint_{0}^{t} A(\eta) d \eta}+\int_{0}^{T}\left[\int_{0}^{s} K(s, \tau) F\left(s, \tau, \hat{x}\left(\tau, x_{0}\right)\right) d \tau+f(s)\right] d t\right] d s
\end{aligned}
$$


Now we prove that $\hat{x}\left(t, x_{0}\right)=x\left(t, x_{0}\right)$ for $x_{0} \in D_{f}$, by proving the following inequality:

$\left\|\hat{x}\left(t, x_{0}\right)-x_{m}\left(t, x_{0}\right)\right\| \leq \Lambda^{m}\left(M_{1}^{*} \frac{T}{2}+\beta\right)$

where $M_{1}^{*}=Q[H R T+N], \quad R=\max _{t \in[0, T]}\|F(s, t, \hat{x})\|$.

let $\mathrm{m}=0$ in (2.1) and from (2.11) we find:

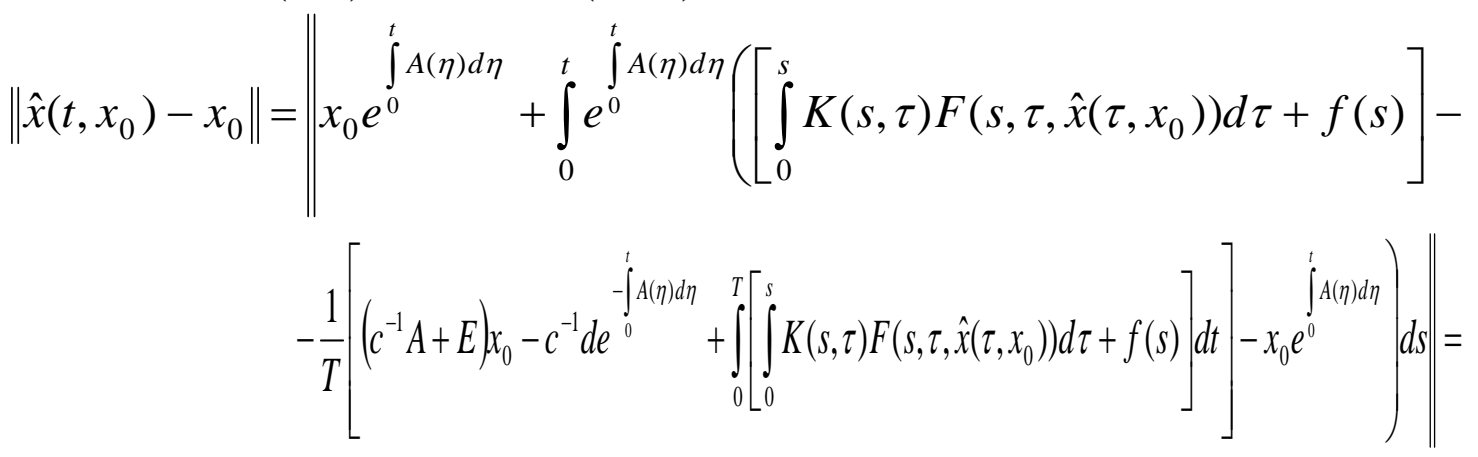
$\leq\left\|\left(1-\frac{t}{T}\right)_{0}^{t} \int_{0}^{t} e^{0} A(\eta) d \eta\left[\int_{0}^{s} K(s, \tau) F\left(s, \tau, \hat{x}\left(\tau, x_{0}\right)\right) d \tau+f(s)\right] d s\right\|+\left\|\frac{t}{T} e^{0} A(\eta) d \eta\left[\left(c^{-1} A+E\right) x_{0}-c^{-1} d e^{-\int^{t} A(\eta) d \eta}\right]\right\|+$ $+\left\|\frac{t}{T} \int_{t}^{T} \int^{\int^{0} A(\eta) d \eta}\left[\int_{0}^{s} K(s, \tau) F\left(s, \tau, \hat{x}\left(\tau, x_{0}\right)\right) d \tau+f(s)\right] d s\right\| \leq$ $\leq 2 t\left(1-\frac{t}{T}\right) Q[H R T+N]+\frac{t}{T} Q\left[\left(c^{-1} A+E\right) x_{0}-c^{-1} d Q^{-1}\right]$ $=\alpha(t) M_{1}^{*}+\beta$ $\left\|\hat{x}\left(t, x_{0}\right)-x_{0}\right\| \leq \alpha(t) M_{1}^{*}+\beta \leq M_{1}^{*} \frac{T}{2}+\beta$

and when $\mathrm{m}=1$ in (2.1) and from (2.11) we find: $\begin{aligned} &\left\|\hat{x}\left(t, x_{0}\right)-x_{1}\left(t, x_{0}\right)\right\|\left\|\left(1-\frac{t}{T}\right) \int_{0}^{t} e^{t} A(\eta) d \eta\left[\int_{0}^{s} K(s, \tau)\left(F\left(s, \tau, \hat{x}\left(\tau, x_{0}\right)\right)-F\left(s, \tau, x_{0}\left(\tau, x_{0}\right)\right)\right) d \tau\right] d s\right\|+ \\ &+\left\|\frac{t}{T} \int_{t}^{T} e^{t} A(\eta) d \eta\left[\int_{0}^{s} K(s, \tau)\left(F\left(s, \tau, \hat{x}\left(\tau, x_{0}\right)\right)-F\left(s, \tau, x_{0}\left(\tau, x_{0}\right)\right)\right) d \tau\right] d s\right\| \\ & \leq\left(1-\frac{t}{T}\right) \int_{0}^{t} Q\left[H L T\left(\alpha(t) M_{1}^{*}+\beta\right)\right] d s+\frac{t}{T} \int_{t}^{T} Q\left[H L T\left(\alpha(t) M_{1}^{*}+\beta\right)\right] d s\end{aligned}$ 


$$
\begin{aligned}
& \leq \frac{T}{2}(Q H L T)\left(\alpha(t) M_{1}^{*}+\beta\right) \\
& =\Lambda\left(\alpha(t) M_{1}^{*}+\beta\right) \\
\| \hat{x}\left(t, x_{0}\right)- & x_{1}\left(t, x_{0}\right) \| \leq \Lambda\left(M_{1}^{*} \frac{T}{2}+\beta\right)
\end{aligned}
$$

and when $m=2$ in (2.1) and from (2.11) we find:

$$
\begin{aligned}
& \left\|\hat{x}\left(t, x_{0}\right)-x_{2}\left(t, x_{0}\right)\right\|=\| x_{0} e^{\int^{t} A(\eta) d \eta}+\int_{0}^{t} e^{t} A(\eta) d \eta\left(\left[\int_{0}^{s} K(s, \tau) F\left(s, \tau, \hat{x}\left(\tau, x_{0}\right)\right) d \tau+f(s)\right]-\right. \\
& -\frac{1}{T}\left[\left(c^{-1} A+E\right) x_{0}-c^{-1} d e^{-\int_{0}^{t} A(\eta) d \eta}+\int_{0}^{T}\left[\int_{0}^{s} K(s, \tau) F\left(s, \tau, \hat{x}\left(\tau, x_{0}\right)\right) d \tau+f(s)\right] d t\right] d s-
\end{aligned}
$$

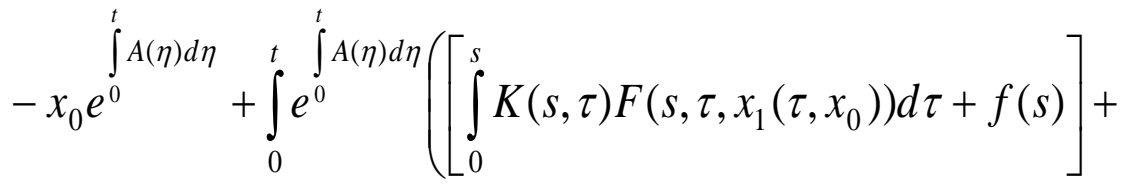

$$
\begin{aligned}
& +\frac{1}{T}\left[\left(c^{-1} A+E\right) x_{0}-c^{-1} d e^{-\int_{0}^{t} A(\eta) d \eta}+\int_{0}^{T}\left[\int_{0}^{s} K(s, \tau) F\left(s, \tau, x_{1}\left(\tau, x_{0}\right)\right) d \tau+f(s)\right] d t\right] d s \| \\
& \leq\left(1-\frac{t}{T}\right) \int_{0}^{t} Q\left[\operatorname{HLT\Lambda }\left(M_{1}^{*} \frac{T}{2}+\beta\right)\right] d s+\frac{t}{T} \int_{t}^{T} Q\left[\operatorname{HLT\Lambda }\left(M_{1}^{*} \frac{T}{2}+\beta\right)\right] d s \\
& \leq \frac{T}{2}(Q H L T) \Lambda\left(M_{1}^{*} \frac{T}{2}+\beta\right) \\
& =\Lambda^{2}\left(M_{1}^{*} \frac{T}{2}+\beta\right) \\
& \left\|\hat{x}\left(t, x_{0}\right)-x_{2}\left(t, x_{0}\right)\right\| \leq \Lambda^{2}\left(M_{1}^{*} \frac{T}{2}+\beta\right)
\end{aligned}
$$

we find that the inequality (2.12) is satisfying when $m=0,1,2$.

Suppose that the inequality (2.12) is satisfying when $m=p$ as the following inequality:

$$
\left\|\hat{x}\left(t, x_{0}\right)-x_{p}\left(t, x_{0}\right)\right\| \leq \Lambda^{p}\left(M_{1}^{*} \frac{T}{2}+\beta\right)
$$

Next we will proof the following inequality: 


$$
\left\|\hat{x}\left(t, x_{0}\right)-x_{p+1}\left(t, x_{0}\right)\right\| \leq \Lambda^{p+1}\left(M_{1}^{*} \frac{T}{2}+\beta\right)
$$

Now

$$
\begin{aligned}
& \left\|\hat{x}\left(t, x_{0}\right)-x_{p+1}\left(t, x_{0}\right)\right\|=\| x_{0} e^{\int_{0}^{t} A(\eta) d \eta}+\int_{0}^{t} e^{t} A(\eta) d \eta\left(\left[\int_{0}^{s} K(s, \tau) F\left(s, \tau, \hat{x}\left(\tau, x_{0}\right)\right) d \tau+f(s)\right]-\right. \\
& -\frac{1}{T}\left[\left(c^{-1} A+E\right) x_{0}-c^{-1} d e^{-\int_{0}^{t} A(\eta) d \eta}+\int_{0}^{T}\left[\int_{0}^{s} K(s, \tau) F\left(s, \tau, \hat{x}\left(\tau, x_{0}\right)\right) d \tau+f(s)\right] d t\right] d s-
\end{aligned}
$$

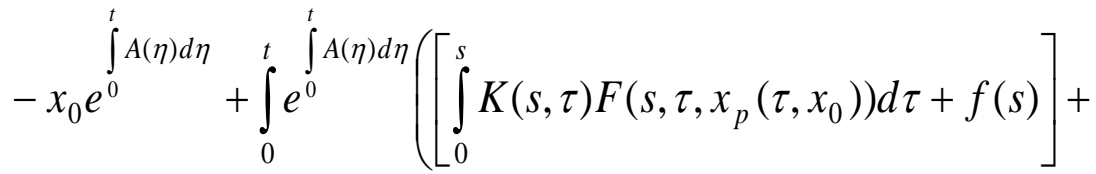

$$
\begin{aligned}
& +\frac{1}{T}\left[\left(c^{-1} A+E\right) x_{0}-c^{-1} d e^{-\int_{0}^{t} A(\eta) d \eta}+\int_{0}^{T}\left[\int_{0}^{s} K(s, \tau) F\left(s, \tau, x_{p}\left(\tau, x_{0}\right)\right) d \tau+f(s)\right] d t\right] d s \| \\
& \leq \frac{T}{2}(Q H L T) \Lambda^{p}\left(M_{1}^{*} \frac{T}{2}+\beta\right)
\end{aligned}
$$

then

$$
\left\|\hat{x}\left(t, x_{0}\right)-x_{p+1}\left(t, x_{0}\right)\right\| \leq \Lambda^{p+1}\left(M_{1}^{*} \frac{T}{2}+\beta\right)
$$

Thus we find that the inequality $(2.15)$ is satisfying when $m=0,1,2, \ldots$.

From the conditions (1.9), (2.10) we get:

$$
\hat{x}\left(t, x_{0}\right)=\operatorname{Lim}_{m \rightarrow \infty} x_{m}\left(t, x_{0}\right)=x\left(t, x_{0}\right) \text {. }
$$

\section{Existence of solution}

The problem of existence solution of the problem (1.1), (1.2) is uniquely connected with the existence of zeros of the function $\Delta=\Delta\left(x_{0}\right)$ which has the form:

$\Delta\left(x_{0}\right)=\frac{1}{T} e^{\int^{t} A(\eta) d \eta}\left[\left(c^{-1} A+E\right) x_{0}-c^{-1} d e^{-\int_{0}^{t} A(\eta) d \eta}+\int_{0}^{T}\left[\int_{0}^{s} K(s, \tau) F\left(s, \tau, x\left(\tau, x_{0}\right)\right) d \tau+f(s)\right] d t\right]$ 
Since this functions are approximately determined from the sequence of functions:

$\Delta_{m}\left(x_{0}\right)=\frac{1}{T} e^{\int^{0} A(\eta) d \eta}\left[\left(c^{-1} A+E\right) x_{0}-c^{-1} d e^{-\int_{0}^{t} A(\eta) d \eta}+\int_{0}^{T}\left[\int_{0}^{s} K(s, \tau) F\left(s, \tau, x_{m}\left(\tau, x_{0}\right)\right) d \tau+f(s)\right] d t\right]$

for $\mathrm{m}=0,1,2, \ldots$.

\section{Theorem 2}

Let all assumptions and conditions of theorem 1 be given, then the following inequality

$$
\left\|\Delta\left(x_{0}\right)-\Delta_{m}\left(x_{0}\right)\right\| \leq \Lambda^{m+1}\left(M_{1}+\frac{2}{T} \beta\right)
$$

satisfies for all $m \geq 0$ and $x_{0} \in D_{f}$.

\section{Proof:}

By (3.1) and (3.2) we get:

$$
\begin{aligned}
& \left\|\Delta\left(x_{0}\right)-\Delta_{m}\left(x_{0}\right)\right\|=\| \frac{1}{T} e^{0} A(\eta) d \eta d\left(\left(c^{-1} A+E\right) x_{0}-c^{-1} d e^{-\int_{0}^{t} A(\eta) d \eta}+\int_{0}^{T}\left[\int_{0}^{s} K(s, \tau) F\left(s, \tau, x\left(\tau, x_{0}\right)\right) d \tau+f(s)\right] d t\right]- \\
& -\frac{1}{T} e^{\int^{0} A(\eta) d \eta}\left[\left(c^{-1} A+E\right) x_{0}-c^{-1} d e^{-\int A(\eta) d \eta}+\int_{0}^{T}\left[\int_{0}^{s} K(s, \tau) F\left(s, \tau, x_{m}\left(\tau, x_{0}\right)\right) d \tau+f(s)\right] d t\right] \\
& \leq \frac{1}{T} \int_{0}^{T}\left\|\int^{\int^{0} A(\eta) d \eta}\right\|\left[\int_{\int}^{s}\|K(s, \tau)\| F\left(s, \tau, x\left(\tau, x_{0}\right)\right)-F\left(s, \tau, x_{m}\left(\tau, x_{0}\right)\right) \| d \tau\right] d t \\
& \leq \frac{1}{T} \int_{0}^{T} Q\left[H L S\left\|x\left(\tau, x_{0}\right)-x_{m}\left(\tau, x_{0}\right)\right\|\right] d t
\end{aligned}
$$

By (2.5) we find

$$
\begin{aligned}
& \leq \frac{1}{T} \int_{0}^{T} Q H L T\left[\Lambda^{m}\left(M_{1} \frac{T}{2}+\beta\right)\right] d t \\
& =\Lambda^{m+1}\left(M_{1}+\frac{2}{T} \beta\right)
\end{aligned}
$$

then

$$
\left\|\Delta\left(x_{0}\right)-\Delta_{m}\left(x_{0}\right)\right\| \leq \Lambda^{m+1}\left(M_{1}+\frac{2}{T} \beta\right)
$$

for all $\mathrm{m}=0,1,2, \ldots$ 


\section{Theorem 3}

If the function $\Delta\left(x_{0}\right)$ is defined by:

$$
\Delta: D_{f} \rightarrow R^{n} \text {, }
$$$$
\Delta\left(x_{0}\right)=\frac{1}{T} e^{\int^{0} A(\eta) d \eta}\left[\left(c^{-1} A+E\right) x_{0}-c^{-1} d e^{-\int_{0}^{t} A(\eta) d \eta}+\int_{0}^{T}\left[\int_{0}^{s} K(s, \tau) F\left(s, \tau, x\left(\tau, x_{0}\right)\right) d \tau+f(s)\right] d t\right]
$$

where the function $x\left(t, x_{0}\right)$ is limit of function (2.1) then the inequalities:

$$
\left\|\Delta\left(x_{0}\right)\right\| \leq M_{1}+\frac{\beta}{T}
$$

where $M_{1}=Q[H M T+N], \beta=\frac{t}{T} Q\left[\left(c^{-1} A+E\right) x_{0}-c^{-1} d Q^{-1}\right]$.

$$
\left\|\Delta\left(x_{0}^{1}\right)-\Delta\left(x_{0}^{2}\right)\right\| \leq\left[\left(c^{-1} A+E\right)+\frac{2}{T} \Lambda A c^{-1}\right] \frac{1}{T}\left\|x_{0}^{1}-x_{0}^{2}\right\| Q
$$

for $x_{0}, x_{0}^{1}, x_{0}^{2} \in D_{f}$.

Proof:

From the continuity of the function $\Delta\left(x_{0}\right)$,then

$$
\begin{aligned}
& \left\|\Delta\left(x_{0}\right)\right\|=\left\|\frac{1}{T} e^{\int^{0} A(\eta) d \eta}\left[\left(c^{-1} A+E\right) x_{0}-c^{-1} d e^{-\int_{0}^{1} A(\eta) d \eta}+\int_{0}^{T}\left[\int_{0}^{s} K(s, \tau) F\left(s, \tau, x\left(\tau, x_{0}\right)\right) d \tau+f(s)\right] d t\right]\right\| \\
& \leq \frac{1}{T} Q\left[\left(c^{-1} A+E\right) x_{0}-c^{-1} d Q^{-1}\right]+\frac{1}{T} Q \int_{0}^{T}\left[\int_{0}^{s} H M d \tau+N\right] d t \\
& \leq \frac{\beta}{T}+\frac{1}{T} \int_{0}^{T} Q[H M T+N] d t \\
& =\frac{\beta}{T}+M_{1} \\
& \left\|\Delta\left(x_{0}\right)\right\| \leq M_{1}+\frac{\beta}{T} .
\end{aligned}
$$

Now from (3.4) we get:

$$
\begin{aligned}
\left\|\Delta\left(x_{0}^{1}\right)-\Delta\left(x_{0}^{2}\right)\right\|= & \| \frac{1}{T} e^{\int^{0} A(\eta) d \eta}\left[\left(c^{-1} A+E\right) x_{0}^{1}-c^{-1} d e^{-\int_{0}^{t} A(\eta) d \eta}+\int_{0}^{T}\left[\int_{0}^{s} K(s, \tau) F\left(s, \tau, x\left(\tau, x_{0}^{1}\right)\right) d \tau+f(s)\right] d t\right]- \\
& -\frac{1}{T} e^{\int^{0} A(\eta) d \eta}\left[\left(c^{-1} A+E\right) x_{0}^{2}-c^{-1} d e^{-\int_{0}^{t} A(\eta) d \eta}+\int_{0}^{T}\left[\int_{0}^{s} K(s, \tau) F\left(s, \tau, x\left(\tau, x_{0}^{2}\right)\right) d \tau+f(s)\right] d t\right]
\end{aligned}
$$




$$
\begin{aligned}
& \leq \frac{1}{T}\left\|\int^{t} A(\eta) d \eta\right\|\left(e^{0} A+E\right)\left\|x_{0}^{1}-x_{0}^{2}\right\|+\frac{1}{T} \int_{0}^{T}\left\|\int^{t} A(\eta) d \eta\right\|\left[\int^{s}\|K(s, \tau)\| F\left(s, \tau, x\left(\tau, x_{0}^{1}\right)\right)-F\left(s, \tau, x\left(\tau, x_{0}^{2}\right)\right) \| d \tau\right] d t \\
& \leq \frac{1}{T} Q\left(c^{-1} A+E\right)\left\|x_{0}^{1}-x_{0}^{2}\right\|+\frac{1}{T} \int_{0}^{T} Q\left[\int_{0}^{s} H L\left\|x\left(\tau, x_{0}^{1}\right)-x\left(\tau, x_{0}^{2}\right)\right\| d \tau\right] d t \\
& \leq \frac{1}{T} Q\left(c^{-1} A+E\right)\left\|x_{0}^{1}-x_{0}^{2}\right\|+\frac{2}{T} Q H L T \frac{T}{2}\left\|x\left(t, x_{0}^{1}\right)-x\left(t, x_{0}^{2}\right)\right\| \\
& =\frac{1}{T} Q\left(c^{-1} A+E\right)\left\|x_{0}^{1}-x_{0}^{2}\right\|+\frac{2}{T} \Lambda\left\|x\left(t, x_{0}^{1}\right)-x\left(t, x_{0}^{2}\right)\right\|
\end{aligned}
$$

then

$$
\left\|\Delta\left(x_{0}^{1}\right)-\Delta\left(x_{0}^{2}\right)\right\| \leq \frac{1}{T} Q\left(c^{-1} A+E\right)\left\|x_{0}^{1}-x_{0}^{2}\right\|+\frac{2}{T} \Lambda\left\|x\left(t, x_{0}^{1}\right)-x\left(t, x_{0}^{2}\right)\right\|
$$

Since the functions $x\left(t, x_{0}^{1}\right), x\left(t, x_{0}^{2}\right)$ are the solution of integral equation:

$$
\begin{aligned}
& x\left(t, x_{0}^{\mu}\right)=x_{0}^{\mu} e^{\int_{0}^{t} A(\eta) d \eta}+\int_{0}^{t} e^{t} A(\eta) d \eta\left(\left[\int_{0}^{s} K(s, \tau) F\left(s, \tau, x\left(\tau, x_{0}^{\mu}\right)\right) d \tau+f(s)\right]-\right. \\
& -\frac{1}{T}\left[\left(c^{-1} A+E\right) x_{0}^{\mu}-c^{-1} d e^{-\int_{0}^{t} A(\eta) d \eta}+\int_{0}^{T}\left[\int_{0}^{s} K(s, \tau) F\left(s, \tau, x\left(\tau, x_{0}^{\mu}\right)\right) d \tau+f(s)\right] d t\right] d s
\end{aligned}
$$

where $\mu=1,2$.

Then by (3.8) and lemma 1.1, we get:

$$
\begin{aligned}
& \left\|x\left(t, x_{0}^{1}\right)-x\left(t, x_{0}^{2}\right)\right\|=\| x_{0}^{1} e^{\int^{t} A(\eta) d \eta}+\int_{0}^{t} e^{t} A(\eta) d \eta\left(\left[\int_{0}^{s} K(s, \tau) F\left(s, \tau, x\left(\tau, x_{0}^{1}\right)\right) d \tau+f(s)\right]-\right. \\
& -\frac{1}{T}\left[\left(c^{-1} A+E\right) x_{0}^{1}-c^{-1} d e^{-\int A(\eta) d \eta}+\int_{0}^{T}\left[\int_{0}^{s} K(s, \tau) F\left(s, \tau, x\left(\tau, x_{0}^{1}\right)\right) d \tau+f(s)\right] d t\right] d s- \\
& -x_{0}^{2} e^{\int^{t} A(\eta) d \eta}+\int_{0}^{t} e^{t} \int^{t} A(\eta) d \eta\left(\left[\int_{0}^{s} K(s, \tau) F\left(s, \tau, x\left(\tau, x_{0}^{2}\right)\right) d \tau+f(s)\right]+\right. \\
& +\frac{1}{T}\left[\left(c^{-1} A+E\right) x_{0}^{2}-c^{-1} d e^{-\int_{0}^{t} A(\eta) d \eta}+\int_{0}^{T}\left[\int_{0}^{s} K(s, \tau) F\left(s, \tau, x\left(\tau, x_{0}^{2}\right)\right) d \tau+f(s)\right] d t\right] d s
\end{aligned}
$$




$$
\begin{aligned}
& \leq \frac{A}{T c}\left\|x_{0}^{1}-x_{0}^{2}\right\| e^{\int^{0} A(\eta) d \eta}+\left\|\left(1-\frac{t}{T}\right) \int_{0}^{t} e^{t} A(\eta) d \eta\left[\int_{0}^{s} K(s, \tau)\left(F\left(s, \tau, x\left(\tau, x_{0}^{1}\right)\right)-F\left(s, \tau, x\left(\tau, x_{0}^{2}\right)\right)\right) d \tau\right] d s\right\|+ \\
& +\left\|\frac{t}{T} \int_{t}^{T} e^{t} e^{0} A(\eta) d \eta\left[\int_{0}^{s} K(s, \tau)\left(F\left(s, \tau, x\left(\tau, x_{0}^{1}\right)\right)-F\left(s, \tau, x\left(\tau, x_{0}^{2}\right)\right)\right) d \tau\right] d s\right\| \\
& \leq \frac{A}{T c}\left\|x_{0}^{1}-x_{0}^{2}\right\| Q+\left(1-\frac{t}{T}\right) \int_{0}^{t} Q\left[\int_{0}^{s} H L\left\|x\left(\tau, x_{0}^{1}\right)-x\left(\tau, x_{0}^{2}\right)\right\| d \tau\right] d s+ \\
& \quad+\frac{t}{T} \int_{t}^{T} Q\left[\int_{0}^{s} H L\left\|x\left(\tau, x_{0}^{1}\right)-x\left(\tau, x_{0}^{2}\right)\right\| d \tau\right] d s \\
& \leq \frac{A}{T c}\left\|x_{0}^{1}-x_{0}^{2}\right\| Q+\left(1-\frac{t}{T}\right) t(Q H L T)\left\|x\left(t, x_{0}^{1}\right)-x\left(t, x_{0}^{2}\right)\right\|+ \\
& \quad+\frac{t}{T}(T-t)(Q H L T)\left\|x\left(t, x_{0}^{1}\right)-x\left(t, x_{0}^{2}\right)\right\| \\
& \leq \frac{A}{T c}\left\|x_{0}^{1}-x_{0}^{2}\right\| Q+\frac{T}{2}(Q H L T)\left\|x\left(t, x_{0}^{1}\right)-x\left(t, x_{0}^{2}\right)\right\|
\end{aligned}
$$

then

$$
\begin{aligned}
& \left\|x\left(t, x_{0}^{1}\right)-x\left(t, x_{0}^{2}\right)\right\| \leq \frac{A}{T c}\left\|x_{0}^{1}-x_{0}^{2}\right\| Q+\Lambda\left\|x\left(t, x_{0}^{1}\right)-x\left(t, x_{0}^{2}\right)\right\| \\
& \left\|x\left(t, x_{0}^{1}\right)-x\left(t, x_{0}^{2}\right)\right\|-\Lambda\left\|x\left(t, x_{0}^{1}\right)-x\left(t, x_{0}^{2}\right)\right\| \leq \frac{A}{T c}\left\|x_{0}^{1}-x_{0}^{2}\right\| Q \\
& (1-\Lambda)\left\|x\left(t, x_{0}^{1}\right)-x\left(t, x_{0}^{2}\right)\right\| \leq \frac{A}{T c}\left\|x_{0}^{1}-x_{0}^{2}\right\| Q \\
& \left\|x\left(t, x_{0}^{1}\right)-x\left(t, x_{0}^{2}\right)\right\| \leq \frac{A}{T c}\left\|x_{0}^{1}-x_{0}^{2}\right\| Q
\end{aligned}
$$

Substituting (3.9) in (3.7) we get (3.6):

$$
\begin{aligned}
& \left\|\Delta\left(x_{0}^{1}\right)-\Delta\left(x_{0}^{2}\right)\right\| \leq \frac{1}{T} Q\left(c^{-1} A+E\right)\left\|x_{0}^{1}-x_{0}^{2}\right\|+\frac{2}{T} \Lambda\left\|x\left(t, x_{0}^{1}\right)-x\left(t, x_{0}^{2}\right)\right\| \\
& \left\|\Delta\left(x_{0}^{1}\right)-\Delta\left(x_{0}^{2}\right)\right\| \leq \frac{1}{T} Q\left(c^{-1} A+E\right)\left\|x_{0}^{1}-x_{0}^{2}\right\|+\frac{2}{T} \Lambda \frac{A}{T c}\left\|x_{0}^{1}-x_{0}^{2}\right\| Q \\
& \left\|\Delta\left(x_{0}^{1}\right)-\Delta\left(x_{0}^{2}\right)\right\| \leq\left[\left(c^{-1} A+E\right)+\frac{2}{T} \Lambda A c^{-1}\right] \frac{1}{T}\left\|x_{0}^{1}-x_{0}^{2}\right\| Q
\end{aligned}
$$




\section{Remark 2.1[4].}

The theorem 3 ensures the stability solution of the system (1.1), when there is a slight change in the point $x_{0}$ accompanied with a noticeable change in the function $\Delta=\Delta\left(t, x_{0}\right)$.

\section{REFERENCES}

1) Butris, R. N. and Ghada Shuker Jameel "Periodic Solutions for Nonlinear System of Integro-Differential Equations with Boundary Integral Condition", International Journal of Mathematical Archive4(10), (2013), 1-14.

2) Ghada Shuker Jameel "Periodic solutions for some nonlinear systems of integro-differential equations of Volterra type", M. Sc. Thesis, University of Mosul, (2005).

3) Jihed Ben Slimene and Joël Blot "Reducibility of systems and existence of solutions for almost periodic differential equations", San Marcos, Texas State University, Electronic Journal of differential equations, Vol.2012(2012), No. 75, pp.1-17.

4) Martynuk, C. B. "The investigation of solution for differential equations with boundary conditions", Ukrain, Kiev, Nauk, Nonlinear problems in the theory of differential equations, No.2, (1991).

5) Samoilenko, A. M. and Ronto, N. I. "A numerical-analytic methods for investigations solution with boundary conditions", Ukrain, Kiev, (1985). 